\title{
EVALUATION OF THYROID NODULES USING THYROID PEROXIDASE AND GALECTIN-3:AN IMMUNOHISTOCHEMICAL STUDY
}

\author{
El-kateb M. I. , Magdy I. Ahmed, Samah S. Mohammed and Mona S.Mostafa \\ Department of Pathology, Faculty of Medicine - Zagazig University
}

\begin{abstract}
Background and aim: Thyroid gland carcinoma is a very prevalent neoplasia worldwide. The majority of malignant and benign lesions of the thyroid can be cytologically established. However, to distinguish between malignant and benign lesions histological demonstration is often required for a precise diagnosis. The aim of this study is to detect the ability of thyroid peroxidase (TPO) and Galectin-3 (Gal-3) to differentiate between thyroid malignant and non-malignant thyroid nodules.

Methods: 50 formalin-fixed, paraffin embedded tissue blocks of different thyroid lesions were previously diagnosed and were sectioned at 3-4 $\mu$ thick and stained with hematoxylin and eosin (H\&E) stain to confirm the histopathologic diagnosis, assess the subtypes of the lesions and assess the histopathologic grading and then were subjected to galectin-3 (Gal-3) and thyroid peroxidase (TPO) immunostaining.

Results: In this retrospective histologic study, galectin-3 had a sensitivity of $93.3 \%$ for papillary thyroid carcinoma and $50 \%$ for follicular thyroid carcinoma. Thyroid peroxidase (TPO) had a sensitivity of $60 \%$ for papillary and $12.5 \%$ for follicular carcinoma. The combination of galectin-3 and TPO had a sensitivity of $100 \%$ for papillary and $50 \%$ for follicular carcinoma.

Conclusion: This study confirms previous observations that galectin-3 alone is highly sensitive for papillary carcinoma but not adequately sensitive for follicular carcinoma. The combination of galectin-3 and TPO is complementary as a diagnostic and prognostic tool for patients with papillary carcinoma.

Key words: Thyroid peroxidase; Galectin-3; Thyroid carcinoma; Immunohistochemistry.
\end{abstract}

\section{INTRODUCTION}

T hyroid nodules of various biological natures occur in approximately $10 \%$ of persons, predominantly females. The nodules represent heterogenous group that comprises both non neoplastic and neoplastic lesions with varying biological behavior. Diagnostic difficulties of these nodules are caused by morphological similarities of biologically diverse lesions, such as papillary hyperplasia versus encapsulated papillary carcinoma or hyperplastic versus neoplastic nodule. Moreover, unusual histologic criteria of malignancy, namely capsular and/or vascular invasion of minimally invasive follicular carcinoma and nuclear characteristics of papillary carcinoma, make differential diagnosis challenging. Difficulties in the interpretation of histological criteria may lead to interobserver variability among pathologists in discriminating between minimally invasive follicular carcinoma and follicular adenoma. These lesions may pose a diagnostic challenge even to more experienced pathologists. Precise pathological diagnosis is essential to optimal treatment of thyroid tumors (1).

Thyroid carcinoma accounts roughly for $1 \%$ of all malignant diseases in USA and represents about $0.2 \%$ of all cancer deaths. Increasing incidence is due to new diagnostic practices which detect smaller tumors (2). In Egypt, the recorded numbers for incidence and prevalence of thyroid carcinoma were very near to the western records. It represented
$1.2 \%$ of total cancers with a low fatality reaching about $8 \%$. It is the most common endocrine malignancy contributing $87 \%$ of cases. Females predominate with a sex ratio of 2.5: 1 . The median age is 40 years in females and 45 years in males (3). Using ancillary diagnostic markers along with histological examination of thyroid nodules of uncertain biological behavior may be helpful. In this study we used galectin-3 (Gal-3) and thyroid peroxidase (TPO) (4).

Thyroid Peroxidase is a transmembrane protein of $107 \mathrm{kDa}$ containing a heme prosthetic group and it is present on the apical surface of the thyroid follicular cells. It is the primary enzyme involved in thyroid hormone synthesis. Malignant thyroid tumors exhibit an anomaly in Thyroid Peroxidase resulting in lower antibody affinity. This antibody may be useful aid in the differentiation between the benign and malignant thyroid tumors (4).

Galectin-3 (gal3) is a beta-galactosidasebinding polypeptide ( $31 \mathrm{kDa}$ molecular weight), a member of the lectin family. It seems to play an important role in number of biological and pathological processes. It is a regulating component of the cell cycle, it regulates cell-cell and cellmatrix interaction, adhesion (major non-integrin laminin binding protein) and migration, it has a role in inflammation, neoplastic transformation, metastatization and in reparation of the damaged cell. Gal3 is expressed in various tissues and cell 
types (e.g. epithelial cells, cells of the immune system) in which it is localized in the nucleus and/or cytoplasm, the cell surface or in the matrix around the cell. Based on recent investigations, gal3 expression correlates with the invasive and metastatic potential of various tumors (5).

\section{MATERIAL AND METHOD}

A total of 50 cases of different thyroid lesions were available for this study. These specimens were received from the pathology department, Faculty of medicine, Zagazig University, National Cancer Institute in the period from 2009 to 2012 and from the private laboratories. All tissue samples were formalin-fixed and paraffin-embedded. Haematoxylin and eosin (H\&E) stained slides and pathologic reports were reviewed to confirm the diagnosis. The clinicopathologic parameters including age, sex and histologic grade were evaluated.

\section{Immunohistochemical Staining:}

Paraffin sections 3-5 um were deparaffinized in the oven at $56{ }^{\circ} \mathrm{C}$ for 30 minutes, and inserted in xylene for 30 minutes. Tissues were rehydrated in descending grades of alcohol 95\%, $85 \%$, and then $75 \%$ for 5 minutes each. Slides were rinsed with distilled water for 5 minutes. Antigen retrieval was performed by boiling in sodium citrate buffer $(0.001 \mathrm{M}, \mathrm{pH}$ 6) for 15 minutes in microwave. Endogenous peroxidase activity was blocked by incubation with $3 \%$ hydrogen peroxide for 10 minutes. Then rinse with distilled water.

Apply primary antibodies (2-3 drops of the first antibody (Gal-3 mouse monoclonal antibody from Thermo Scientific/Lab Vision Corporation, Fermont, USA, clone:9C4) on each slide to cover the specimen and (2-3 drops of the second antibody (TPO anti mouse monoclonal anti body from USBiological, Massachusetts, USA, clon:11E363) on each slide to cover the specimen overnight at $4^{\circ} \mathrm{C}$. After 3 wash with phosphate buffered saline (PBS), sections were incubated with biotinylated secondary antibodies for $30 \mathrm{~min}$. This is followed by incubation with streptavidin-biotin-peroxidase complex. After 3 rinses with (PBS), the slides were incubated with diaminobenzidine for $15 \mathrm{~min}$. The slides were rinsed with distilled water and counerstained with hematoxylin for 3 minutes. This was followed by washing in cold running water, then wash in distilled water. Sections were dehydrated in ascending grades of alcohol and cleared with xylene, then coverslipped and examined.

\section{Scoring Criteria:}

Immunohistochemical staining scores were assigned by 2 pathologists. The staining intensity was graded as 0 (no staining), $1+$ (slight staining), $2+$ (moderate staining), or $3+$ (intense staining), and the proportion of stained cells was scored as $1+$ $(<5 \%$ of cells $), 2+(5 \%-50 \%$ of cells $)$, or $3+(>50 \%$ of cells).

A positive result for Gal-3 was considered consistent with cancer, and a negative result for TPO was considered consistent with cancer. Sections from a case of papillary carcinoma of the thyroid that previously was characterized as positive for Gal-3 and adjacent benign thyroid tissue that was known to be positive for TPO were used as positive control samples (6).

Sensitivity, specificity, positive predictive value, and negative predictive value were calculated for Gal-3 and TPO individually and for the 2 antibodies in combination. Sensitivity was defined as the number of carcinomas with positive results (positive Gal-3 or negative TPO) as a percentage of the total number of carcinomas. Specificity was defined as the number of benign lesions with negative results as a percentage of the total number of benign lesions. The positive predictive value was defined as the number of carcinomas with positive results as a percentage of the total number of cases with positive results, and the negative predictive value was defined as the number of benign lesions with negative results as a percentage of the total number of cases with negative results.

Statistical analysis: Statistical analysis was performed using SPSS software. The degree of correlation between different parameters was evaluated by using the chi- square test. A difference of $P<0.05$ between groups was considered significant.

\section{Immunostaining}

\section{RESULTS}

Regarding Gal-3 immunoreactivity, of 10 follicular adenomas, 6(60\%) were negative for Gal-3 and $4(40 \%)$ were positive, including the following: 2 Hürthle cell adenoma showing 3+intensity, 1 showing 2+ intensity, and 1 showing 1+ intensity. The studied 2 cases of Hashimoto's thyroiditis showed restricted Gal-3 immunoreactivity to Hürthle cells which were presented focally. The studied 2 cases of lymphocytic thyroiditis and the 3 cases of colloid goiter were negative to Gal-3.

$14(93.3 \%)$ cases out of 15 papillary carcinomas were positive for Gal-3. 12 cases showed strong diffuse staining, 1 had moderate staining and 1 had mild staining and one sample showed no staining. 
Of 8 follicular carcinomas, $4(50 \%)$ were positive for Gal-3, while the remaining 4 follicular carcinomas showed no staining. 2 cases of widely invasive follicular carcinoma showed strong positivity for Gal-3 immunostaining, 1 showed moderate immunostaining and one showed mild immunostaining while 2 cases were negative. The 2 cases of minimally invasive follicular carcinoma showed no staining for Gal-3. There was no significant difference in Gal-3 immunostaining between widely invasive follicular carcinoma and minimally invasive follicular carcinoma $\left(x^{2}=2.67\right.$ and $\mathrm{p}=0.44$ )

The 2 studied cases of anaplastic and the 2 studied cases of poorly differentiated thyroid carcinoma were positive for Gal-3. 2 of the studied 6 cases of medullary thyroid carcinoma showed strong diffuse staining with Gal-3, 2 showed mild to moderate staining and 2 lesions were negative for Gal-3.

There was a statistically significant

relationship between Gal-3 in the studied benign and malignant thyroid lesions ( $\mathrm{x} 2$ $=9.56$ and $\mathrm{p}=0.002 *$ ).

Regarding TPO immunoreactivity, all cases of benign thyroid lesions were strongly and diffusely positive for TPO.

$6(40 \%)$ of 15 papillary carcinomas showed strong diffuse staining for TPO and $9(60 \%)$ sample showed no staining.

Of 8 follicular carcinomas, $7(87.5 \%)$ were positive for TPO. While one case only showed no staining. 5 cases of widely invasive follicular carcinoma showed strong and diffuse positivity for TPO immunostaining while one case only was negative. The 2 cases of minimally invasive follicular carcinoma showed strong and diffuse staining for TPO. There was no significant difference in TPO immunostaining between widely invasive follicular carcinoma and minimally invasive follicular carcinoma $\left(x^{2}=0.89\right.$ and $\mathrm{p}=$ $0.64)$.

The 2 studied cases of anaplastic thyroid carcinoma and the 2 studied cases of poorly differentiated thyroid carcinoma were negative for TPO. 2 cases of the studied 6 cases of medullary thyroid carcinoma showed moderate staining with TPO, 1 case showed strong staining and 3 lesions were negative for TPO.

There was a statistically significant relationship between TPO in the studied benign and malignant thyroid lesions ( $\mathrm{x} 2$

$=13.16$ and $\mathrm{p}=0.001 *)$.
Table (1): shows the immunohistochemistry results of Gal-3 and TPO in malignant thyroid lesions and other nonmalignant thyroid lesions. It was statistically significant.

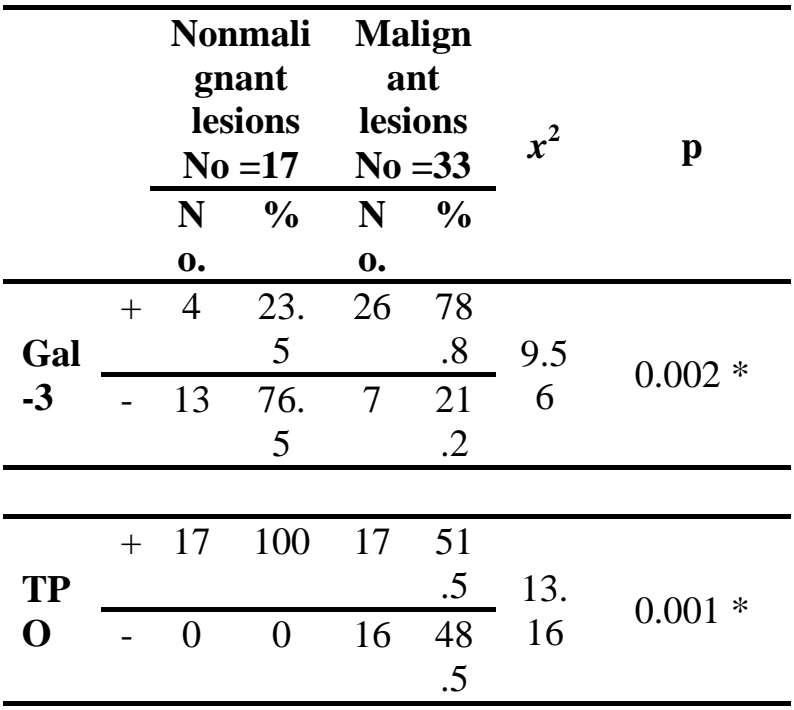

Table (2): Sensitivity, Specificity, Positive and Negative Predictive Values in 50 Cases.

\begin{tabular}{|c|c|c|c|}
\hline & Gal-3 & TPO & $\begin{array}{l}\text { Gal-3 } \\
\text { and } \\
\text { TPO }\end{array}$ \\
\hline \multicolumn{4}{|l|}{ Sensitivity (\%) } \\
\hline $\begin{array}{l}-\quad \text { Any } \\
\text { carcinoma }(n=33)\end{array}$ & $\begin{array}{l}(26) \\
78.8\end{array}$ & $\begin{array}{l}(17) \\
51.5\end{array}$ & $\begin{array}{l}(29) \\
87.8\end{array}$ \\
\hline $\begin{array}{l}\text { Papillary } \\
(\mathrm{n}=15)\end{array}$ & $\begin{array}{l}(14) \\
93.3 \\
\end{array}$ & (9) 60 & $\begin{array}{l}(15) \\
100 \\
\end{array}$ \\
\hline $\begin{array}{ll} & \text { Follicular } \\
(\mathrm{n}=8) & \end{array}$ & (4) 50 & $\begin{array}{c}(1) \\
12.5\end{array}$ & (4) 50 \\
\hline $\begin{array}{ll}- & \text { Medullary } \\
(\mathrm{n}=6) & \\
\end{array}$ & \multirow{3}{*}{ (8) 80} & \multirow{3}{*}{ (7) 70} & \multirow{3}{*}{$\begin{array}{l}(10) \\
100\end{array}$} \\
\hline $\begin{array}{l}\text { Poorly } \\
\operatorname{diff}(n=2)\end{array}$ & & & \\
\hline $\begin{array}{ll}- & \text { Anaplastic } \\
(\mathrm{n}=2) & \\
\end{array}$ & & & \\
\hline Specificity (\%) & $\begin{array}{c}(13 / 17) \\
76.47\end{array}$ & $\begin{array}{c}(17 / 17) \\
100\end{array}$ & $\begin{array}{c}(13 / 17) \\
76.47\end{array}$ \\
\hline $\begin{array}{l}\text { Positive predictive } \\
\text { value }(\%)\end{array}$ & $\begin{array}{c}(26 / 30) \\
86.6 \\
\end{array}$ & $\begin{array}{c}(17 / 17) \\
100 \\
\end{array}$ & $\begin{array}{c}(29 / 33) \\
87.8 \\
\end{array}$ \\
\hline $\begin{array}{l}\text { Negative } \\
\text { predictive value } \\
(\%)\end{array}$ & $\begin{array}{c}(13 / 20) \\
65\end{array}$ & $\begin{array}{c}(17 / 33) \\
51.5\end{array}$ & $\begin{array}{c}(13 / 17) \\
76.47\end{array}$ \\
\hline
\end{tabular}

As shown in table (2) the sensitivity of Gal-3 for the detection of any carcinoma was $78.8 \%$. The 
sensitivity for papillary carcinoma alone was $93.3 \%$, while the sensitivity for follicular carcinoma was $50 \%$ and the sensitivity for the other types of carcinomas was $80 \%$. The specificity was $67.47 \%$. The positive predictive value was $86.6 \%$, and the negative predictive value was $65 \%$.

By using a negative result for the TPO stain as consistent with cancer, the sensitivity for the detection of any carcinoma was $51.5 \%$. The sensitivity for papillary carcinoma alone was $60 \%$, while the sensitivity for follicular carcinoma was $12.5 \% \%$ and the sensitivity for the other types of carcinomas was $70 \%$. By using a positive stain for TPO as negative or not suggestive of cancer, the specificity was $100 \%$. The positive predictive value also was $100 \%$, and the negative predictive value was $51.5 \%$. The results of the Gal-3 and TPO immunostaining then were combined. A positive result (consistent with cancer) was considered to be either a positive Gal-3 stain or a negative TPO stain. By using these criteria, 15 of 15 papillary carcinomas were positive, giving a sensitivity of $100 \%$. Four cases of follicular carcinomas were positive, for a sensitivity of $50 \%$ and the sensitivity for the other types of carcinomas was $100 \%$. The overall sensitivity for the detection of any carcinoma was $87.8 \%$. Of 17 benign lesions, 13 had an overall negative combination of stains, giving a specificity of $76.47 \%$. The positive predictive value of the combination was $87.8 \%$, while the negative predictive value was $76.47 \%$.

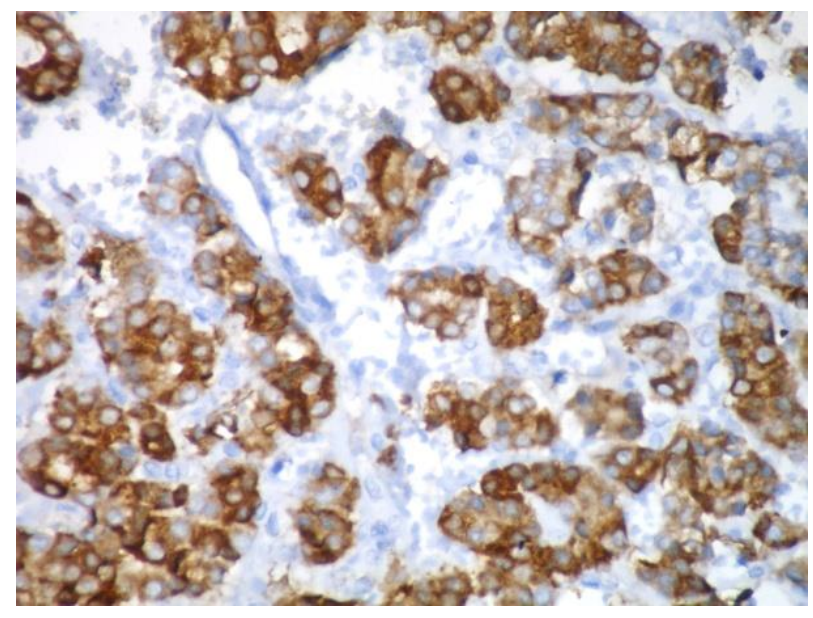

Figure (A): A case of microfollicular adenoma showing strong staining with TPO X400.

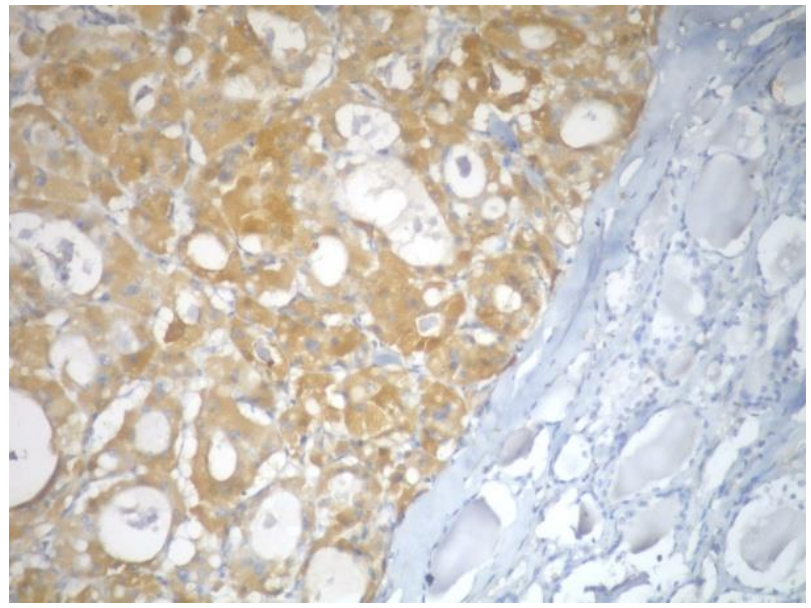

Figure (B): A case of Hürthle cell adenoma showing strong staining with Gal-3, while the normal thyroid tissue is negative for Gal-3 X200.

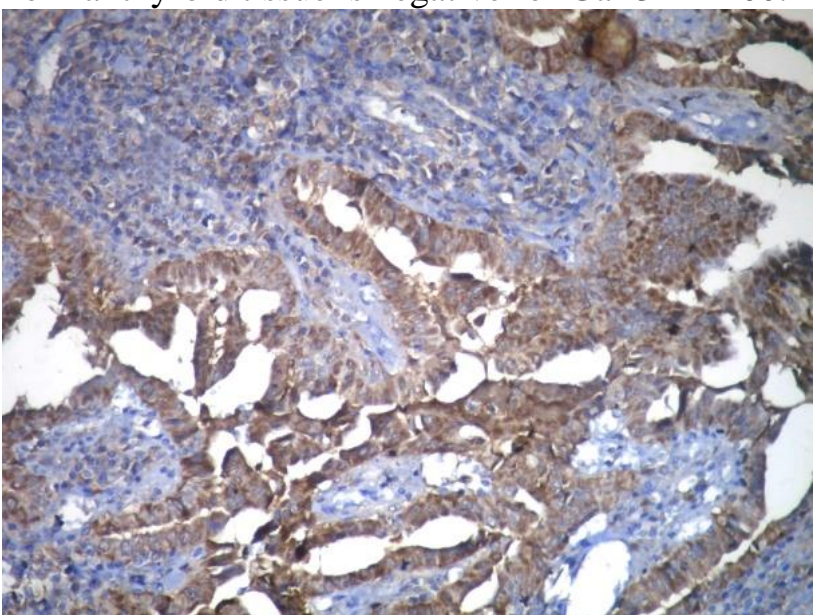

Figure (C): A case of papillary carcinoma showing marked reactivity with Gal-3 X200.

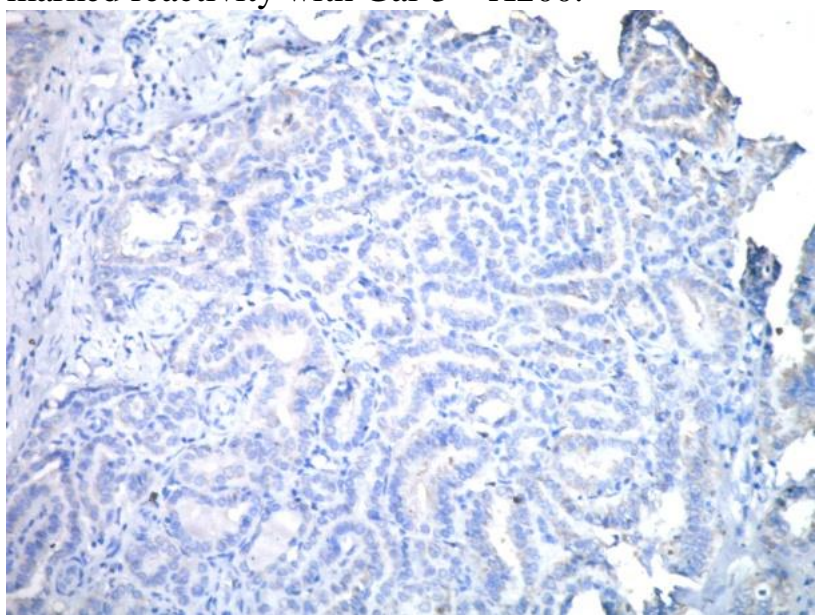

Figure (D): A case of papillary carcinoma showing negative reactivity with TPO X200. 


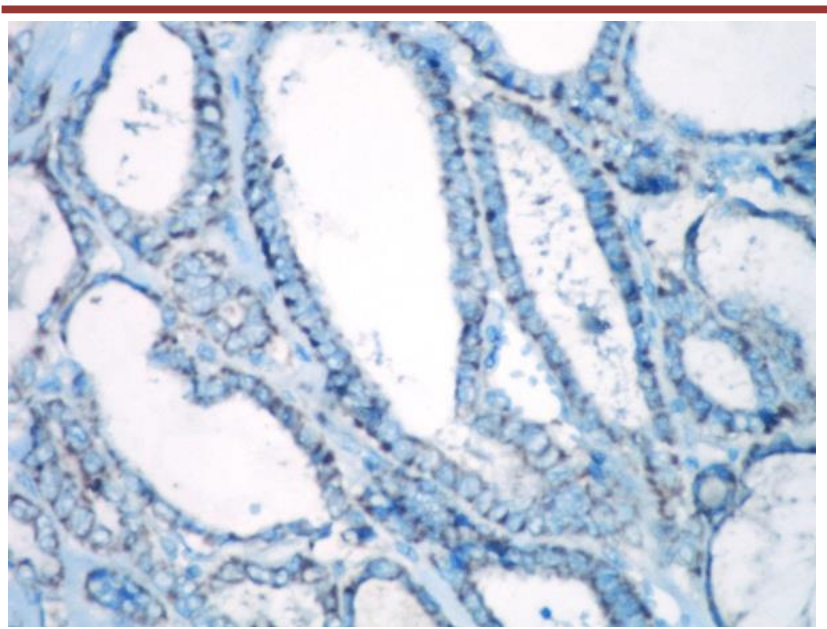

Figure (D): A case of papillary carcinoma follicular variant showing negative reactivity with TPO $\mathrm{X} 400$.

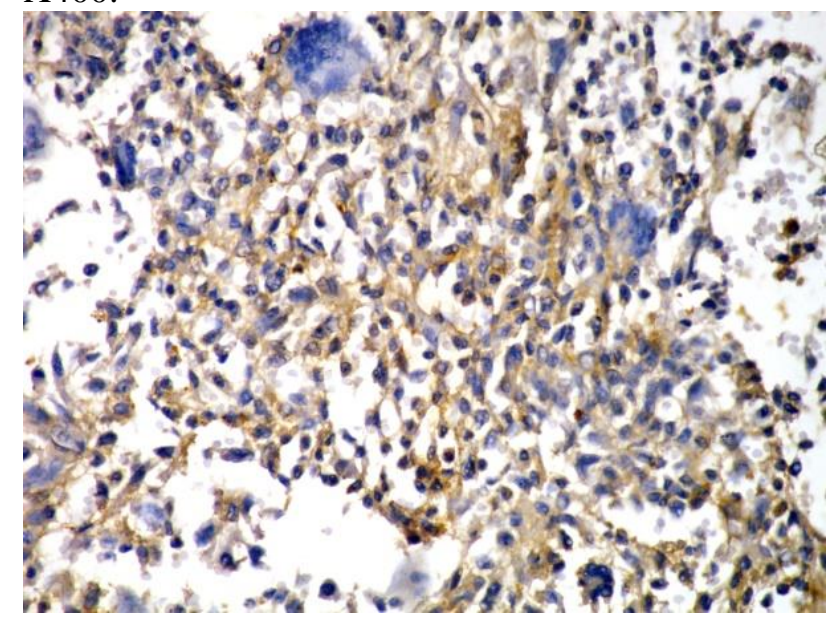

Figure (F): A case of anaplastic carcinoma with spindle cells and giant cells showing moderate Gal3 immunoreactivity X400.

\section{DISCUSSION}

The present work included 50 randomly selected cases of thyroid gland lesions including 17 cases (34\%) of benign thyroid lesions and 33 cases (66\%) of malignant thyroid lesions. These cases after collection of their data were subjected to galectin-3 (Gal-3) and thyroid peroxidase (TPO) immunostaining to detect their accuracy in differentiating between thyroid malignant and nonmalignant thyroid nodules which were removed surgically.

All the parenchymatous background was negative to Gal-3 except in Hashimoto's thyroiditis, where the oncocytes showed positive cytoplasmic staining raising a question on the role of these cells in the development of cancer. Di Pasquale et al.(7) described the pathologic features of papillary carcinoma arising in a background of Hashimoto's thyroiditis and found the presence of atypical nodules (clear nuclei, occasional grooves, no nuclear pseudo-inclusions) that may represent a precursor lesion of papillary carcinoma.

We found that of 10 follicular adenomas, 6 $(60 \%)$ were negative for Gal-3 and $4(40 \%)$ were positive. This is in agreement with Saleh et al. (8) who found $(41.3 \%)$ of their studied follicular adenoma cases stained with Gal-3. This result also is close to the result of Herrmann et al. (9) that had (37\%) of the studied follicular adenoma cases reactive with Gal-3. In a large multicenter trial, Gasbarri et al. (10) and Bartolazzi et al. (11) identified Gal-3 expression in 3\% only of follicular adenoma cases. The high rate of expression of Gal-3 in $72 \%$ of follicular adenoma cases reported by Mehrotra et al. (12).

The studied $2(100 \%)$ cases of Hürthle cell adenoma reacted strongly and diffusely with Gal-3 and this is in agreement of Herrmann et al.(9). Nascimento et al. (13) mentioned that this positivity of Hürthle -cell adenomas can predict that these benign lesions may later become malignant.

The studied 2 cases of Hashimoto's thyroiditis showed restricted Gal-3 immunoreactivity to follicular cells showing oncocytic changes and localized around a lymphoid follicle. The follicular cells entrapped in areas of great chronic lymphocyte inflammation were also positive. This is in agreement with Kova'cs et al. (14) and Herrmann et al. (9).

Rabinovich and Rubinstein (15) stated that the most likely explanation for the Gal3 expression of non-neoplastic follicular cells in an inflamed area is neosynthesis affected by cytokines secreted by inflammatory cells or the simple permeation of Gal3 abundantly shed by lymphocytes into the neighboring follicular cells. Kova'cs et al. (14) mentioned that due to the benign feature of follicular cells, the Gal3 positivity in inflammatory lesions will not create any diagnostic problem; however, it may be problematic in cytological smears especially if the inflammatory elements are not prominent.

In this study the 2 cases of lymphocytic thyroiditis and the 3 cases of colloid goiter were negative to Gal-3 this is in agreement with Fernandez et al. (16), Bartolazzi et al (17), Herrmann et al. (9) and Kova'cs et al. (14), but in disagreement with the reports from Beesely and McLaren (18) and Prasad et al. (19) that found Gal-3 positivity in $38 \%$ and $55 \%$ of goiter specimens, respectively.

In this study 14 (93.3\%) of 15 papillary carcinomas were positive for Gal-3. This result is close to those 
results of Kova'cs et al. (14) whose study gave (95\%) of papillary thyroid carcinoma cases reactive with Gal-3, Weber et al. (6) with $(92 \%)$ of cases positive with Gal-3 and Prasad et al. (19) who found $(94 \%)$ of papillary carcinomas stained with Gal-3. (100\%) of cases were positive in the studies of Fernandez et al. (16) and Saggiorato et al. (20). This work included 8 cases of follicular carcinomas, (50\%) were positive for Gal-3. There was no significant difference in Gal-3 immunostaining between widely invasive follicular carcinoma and minimally invasive follicular carcinoma. This result is similar to that of Fernandez et al. (16) and Torres-Cabala et al. (21). This result is also close to the results of Weber et al. (6) and Sapio et al. (22) with $(44 \%)$ and $(40 \%)$ respectively positive cases of follicular carcinoma with Gal-3. In large multicenter trials, Gal-3 expression ranged from $95 \%$ to $100 \%$ of follicular thyroid carcinoma cases (19), (11), (18). These different results may be due to the larger sample size and the usage of a different Gal-3 antibody from Novocastra Laboratories.

The $2(100 \%)$ studied cases of anaplastic thyroid carcinoma were positive for Gal-3. This is in agree with Fernandez et al.(16) Bartolazzi et al (17), Herrmann et al. (9), Prasad et al. (19) and Saggiorato et al. (20). In an immunohistochemical marker panel study of Wiseman et al. (23). of anaplastic thyroid carcinoma (ATC) and differentiated thyroid carcinoma (DTC), Gal-3 expression was found to be up-regulated in ATC compared with adjacent associated DTC foci, although the change was not statistically significant.

The 2 studied cases of poorly differentiated thyroid carcinoma (PDTC) were positive for Gal-3. This is in agreement with Herrmann et al. (9).

In this study, $(66.6 \%)$ cases of medullary thyroid carcinoma were positive with Gal-3. This is near the result of Kova'cs et al. (14).

As regarding thyroid peroxidase immunohistochemical reactivity in this study all cases of benign thyroid lesions were strongly and diffusely positive for TPO. This is in agreement with Weber et al. (6) and Yousaf et al. (24). The results of $\mathbf{S}$ Savin et al. (25)and Yegen et al.(26)were different, as (60\%) of the follicular adenoma cases were considered positive with TPO and the rest of cases were negative and considered as malignant because they applied the cut-off values proposed by De Micco et al.(27) where lesions were considered positive and hence allegedly benign, if more than $80 \%$ of the thyroid epithelial cells stained for TPO and tissue staining of less than
$80 \%$ of the follicular cells/specimen was considered malignant.

In our study, $(40 \%)$ of papillary carcinomas (PTC) were reactive for TPO and $(60 \%)$ of samples were negative. This was close to the resuls Weber et al. (6) and Yegen et al. (26). This is in contrast with Yousaf et al. (24) who found (100\%) of PTC negative for TPO.

Of 8 follicular carcinomas (FC) $(87.5 \%)$ were positive for TPO, while one case only showed no staining. There was no significant difference in Gal3 immunostaining between widely invasive follicular carcinoma and minimally invasive follicular carcinoma. This result is nearly similar to that of Weber et al. (6) who found (88.8\%) of the FC cases positive for TPO. Savin et al. (25) and Yegen et al. (26) applied the cut-off values of $80 \%$ and their positive FC cases for TPO were $(36.84 \%)$ and $(42 \%)$ respectively. Savin et al. (25) and Yegen et al. (26) also found no relationship between TPO staining and the type of invasion in follicular carcinomas, because the widely invasive type, a highly aggressive malignancy, was not necessarily negative. They therefore failed to establish a significant decrease in TPO protein expression in follicular carcinoma with higher aggressiveness.

In this study the 2 studied cases of anaplastic thyroid carcinoma and the 2 studied cases of poorly differentiated thyroid carcinoma were negative for TPO and this is in agreement with Yousaf et al. (24).

Fifty percent of cases of the studied medullary thyroid carcinoma showed staining with TPO. Yousaf et al. (24) studied one case only of medullary carcinoma and it was negative for TPO.

In this study Gal-3 had high sensitivity $(93.3 \%)$ for the detection of papillary carcinoma. The combination of Gal-3 and TPO also was highly sensitive $(100 \%)$ for the detection of papillary carcinoma. This result is close to the result of Weber et al. (6) and Savin et al. (4). Gal-3 clearly is a useful adjunct to the standard histopathologic diagnosis of papillary thyroid carcinoma.

Neither Gal-3 nor the combination of Gal-3 and TPO was found to have adequate sensitivity for the detection of follicular carcinoma in this study. The results of this study for follicular carcinomas (50\% sensitivity of Gal-3) using the Thermo Scientific/Lab Vision Corporation Gal-3 antibody are more consistent with the results of Herrmann et al. (9), Fernandez et al. (16) and Weber et al. (6) and call into question the usefulness of Gal-3 for the diagnosis of follicular carcinoma. Bartolazzi et al. 
(17) reported (93\%) sensitivity for minimally invasive follicular carcinomas and (100\%) sensitivity for widely invasive follicular carcinomas with GAL-3. Saggiorato et al. (20) similarly found $(100 \%)$ sensitivity for minimally invasive follicular carcinomas with Gal-3. These groups as we mentioned before used a Gal-3 antibody from Novocastra Laboratories.It is, however, important to view any results regarding sensitivity for the detection of follicular carcinoma with some caution, because the diagnosis of this entity is challenging, and it is difficult to be certain of a true gold standard.

The results for TPO from the present study were quite different. Sensitivities between $97 \%$ and $100 \%$ have been reported in multiple studies, (27), (28), (29),(30), while the sensitivity in the present study was only $(51.5 \%)$ overall and $(12.5 \%)$ for follicular carcinomas. This result was close to the results of Weber et al. (6), the overall sensitivity was (39\%) and the sensitivity for follicular carcinomas was only $(11 \%)$. Conversely, the present study reports a specificity of $100 \%$, while the specificity of other multiple studies ranged from $68 \%$ to $90 \%$.The reasons for these discrepancies are unclear (27), (28), (29), (30).

In this study, the sensitivity of Gal-3 in the diagnosis of medullary carcinoma, poorly differentiated carcinoma and anaplastic carcinoma collectively was (80\%) and for TPO was (70\%). Unfortunately, the studies of these two markers together did not focus on these types of cancer, but only focused on papillary carcinoma and follicular carcinoma. So we cannot compare our results with other studies.

The pathophysiology of Gal-3 (a marker indicating malignant transformation) and of TPO (a marker indicating normal thyroid function) suggests that these markers could be useful prognostic tools. The continued expression of TPO in a cancer, for example, might predict a better clinical outcome than the lack of such expression. The observations that Gal-3 might have a role in malignant transformation and metastasis, raises important questions about the basic cell biology of thyroid carcinoma and the role of Gal-3 in it (Weber et al.(6).

\section{CONCLUSION AND RECOMMENDATION}

- Combined immunostaining for gal-3 and TPO may be useful in the diagnosis of differentiated thyroid carcinomas because of improvement of sensitivity in comparison with individual markers.
- Definite conclusions about TPO and gal-3 clinical implications in predicting the outcome of malignant disease must await the collection of data from a large number of clinically correlated specimens analyzed in a prospective study.

- We recommend using Gal-3 as a useful mean to increase the likelihood of detecting malignant tumors. This practical low cost immunohistochemical test of commercially available marker can help to optimize the management of patients with thyroid nodules and reduce unnecessary surgical resection of benign nodules.

- Additional studies are needed toward the quest of identifying useful markers for differentiating benign from malignant thyroid nodules.

\section{Acknowledgments:}

We wish to thank the members of Pathology Department, Faculty of Medicine, Zagazig University and every one helped us to complete this work. Without their continued efforts and support, we would have not been able to bring our work to a successful completion.

\section{REFERENCES}

1) Chung-Che W.; Lyssa F.; Giulia C. et al. (2011): A Large Multicenter Correlation Study of Thyroid Nodule Cytopathology and Histopathology. Thyroid. March, 21(3): 243-51.

2) Maria M.; Bugalho.; Nelson W. et al.(2011) : Thyroid Oncology. J Thyroid Res; 467570.

3) El-Bolkainy N.; Akram N.; and El-Bolkainy T. (2005): Topographic Pathology of Cancer. The Nat Cancer Inst. 3rd edition (11):89-91.

4) Savin S.; Dubravka C.; Tijana I. et al. (2008): Thyroid peroxidase and galectin-3 immunostaining in differentiated thyroid carcinoma with clinicopathologic correlation. Human Pathology. 39, 1656-63.

5) Krzeslak A. and Lipinska A. (2004): Galectin-3 as a multifunctional protein. Cell Mol Biol Lett 9:305-28.

6) Weber B.; Kenneth S.; David H. et al. (2004): The Use of a Combination of Galectin-3 and Thyroid Peroxidase for the Diagnosis and Prognosis of Thyroid Cancer . Am J Clin Pathol .122:524-31

7) Di Pasquale M.; Rothstein L. and Palazzo P. (2001): Pathologic features of Hashimoto's-associated papillary thyroid carcinomas. Hum Pathol. Jan; 32(1):24-30.

8) Saleh A.; Bo J.; John B. et al. (2010): Utility of immunohistochemical markers in differentiating benign from malignant follicular derived thyroid nodules. Diagnostic Pathology. 5:9.

9) Herrmann E.; Virginia L.; Theresa P. et al. (2002): Immunohistochemical Expression of Galectin-3 in 
Benign and Malignant Thyroid Lesions. Arch Pathol Lab Med. June; (126).

10) Gasbarri A.; Martegani P.; Del Prete F. et al (1999): Galectin-3 and CD44v6 isoforms in the preoperative evaluation of thyroid nodules. J Clin Oncol. 17:349402

11) Bartolazzi A.; Orlandi F.; Saggiorato E. et al. (2008): Galectin-3-expression analysis in the surgical selection of follicular thyroid nodules with indeterminate fine-needle aspiration cytology: a prospective multicentre study. Lancet Oncol, 9:54349.

12) Mehrotra P.; Okpokam A.; Bouhaidar R. et al. (2004): Galectin-3 does not reliably distinguish benign from malignant thyroid neoplasms. Histopathology, 45:493-500.

13) Nascimento C.; Bisi H.; Alves A. at al. (2001): Differential reactivity for galectin-3 in Hurthle cell adenomas and carcinomas. Endocrine Pathology J.1: 275-79.

14) Kova'cs B.; Ja'nos F.; Ga'bor W. et al. (2003): The investigation of galectin-3 in diseases of the thyroid gland. European Journal of Endocrinology.149:44953.

15) Rabinovich A. and Rubinstein N. (2001): Galectins: a novel family of proteins involved in the regulation of the immune response. Implications in immunopathological processes. Medicine. 61: 85-92

16) Fernandez L.; Merino J.; Gomez M. et al. (1997): Galectin-3 and laminin expression in neoplastic and non-neoplastic thyroid tissue. J Pathol . 86 (11):180: 6.

17) Bartolazzi A.; Gasbarri A.; Papotti M. et al. (2001): Application of an immunodiagnostic method for improving preoperative diagnosis of nodular thyroid lesions. Lancet Oncol. 357:1644-1650.

18) Beesley F. and McLaren M. (2002): Cytokeratin 19 and galectin-3 immunohistochemistry in the differential diagnosis of solitary thyroid nodules. Histopathology, 41:236-24381.

19) Prasad L.; Pellegata S.; Huang Y. et al. (2005): Galectin-3, fibronectin-1. CITED-1, HBME1 and cytokeratin-19 immunohistochemistry is useful for the differential diagnosis of thyroid tumors. Mod Pathol, 18:48-57.

20) Saggiorato E.; De R.; Volante M. et al. (2005): Characterization of thyroid 'follicular neoplasms' in fine-needle aspiration cytological specimens using a panel of immunohistochemical markers: a proposal for clinical application. Endocr Relat Cancer. 12:30517.

21) Torres-Cabala C.; Bibbo M.; Panizo-Santos A. et al. (2006): Proteomic identification of new biomarkersand application in thyroid cytology. Acta Cytol. 50:518-528.

22) Sapio R.; Guerra A.; Posca D. et al. (2007): Combined analysis of galectin-3 and BRAFV600E improves the accuracy of fine-needle aspiration biopsy with cytological findings suspicious for papillary thyroid carcinoma. Endocr Relat Cancer. 14:1089-97.

23) Wiseman M.; Griffith L.; Deen S. et al. (2007): Identification of molecular markers altered during transformation of differentiated into anaplastic thyroid carcinoma. Arch Surg. 142:717-27.

24) Yousaf U.; Christensent H.; Rasmussen K. et al. (2008): Immunohistochemical staining for thyroid peroxidase (TPO) of needle core biopsies in the diagnosis os scintigraphically cold thyroid nodules. Clinical Endocrinology. 68: 996-1001.

25) Savin S.; Cvejic D.; IsicT. et al. (2006) : The efficacy of the thyroid peroxidase marker FOR distinguishing follicular thyroid carcinoma from follicular adenoma . Exp Oncol. 24:643-49.

26) Yeğen G.; Mehmet İ.; Yeşim N. et al (2009): Follicular Lesions of Thyroid. Turkish Journal of Pathology. 25(3): 90-99.

27) De Micco C.; Zoro P.; Garcia S. et al. (1994): Thyroid peroxidase immunodetection as a tool to assist diagnosis of thyroid nodules on fine-needle aspiration biopsy. Eur J Endocrinol. 131:474-79.

28) Pluot M.; Faroux J.; Flament B. et al. (1996): Quantitative cytology and thyroperoxidase immunochemistry: new tools in evaluating thyroid nodules by fine-needle aspiration. Cancer Detect Prev. 20:285-93.

29) Faroux J.; Theobald S.; Pluot M. et al. (1997): Evaluation of the monoclonal antibody antithyroperoxidase MoAb47 in the diagnostic decision of cold thyroid nodules by fine-needle aspiration. Pathol Res Pract. 193: 705-12.

30) Henry F.; Denizot A.; Porcelli A. et al. (1994): Thyroperoxidasei mmunodetection for the diagnosis of malignancy on fineneedle aspiration of thyroid $\begin{array}{llll}\text { nodules. World J } & \text { Surg. 18:529-3 }\end{array}$ 